\title{
Assessment of Genetic Diversity in Chickpea (Cicer arietinum L.) Germplasm under Normal Sown Condition of Bihar
}

\author{
Tejashwini Agrawal, Anand Kumar*, Sanjay Kumar, Anil Kumar, \\ Manoj Kumar, Satyendra and Sadia Perween
}

Department of Plant Breeding and Genetics, Bihar Agricultural University, Sabour

(Bhagalpur)-813210, India

*Corresponding author

\section{A B S T R A C T}

An experiment was conducted during Rabi 2016-17 at Pulses Research farm, Bihar Agricultural University, Sabour (Bhagalpur) to study the existing genetic diversity in thirty six Desi chickpea genotypes under normal sown condition. All the genotypes were grouped into seven clusters as per Ward minimum variance method revealed the presence of considerable amount of genetic diversity in the material with each other for different yield and yield attributing traits. The cluster II containing the maximum of 13 genotypes followed by 9 genotypes in cluster I, 5 genotypes in cluster III, 3 genotypes each in cluster IV and V, 2 genotypes in cluster in cluster VI and mono-genotypes in cluster. The intracluster $\mathrm{D}^{2}$ value ranged from 0.00 to 421.27 while, inter-cluster $\mathrm{D}$ value ranged from

\section{Keywords}

Chickpea, Genetic diversity, $\mathrm{D}^{2}$ statistics and grain yield

\section{Article Info}

Accepted:

28 March 2018

Available Online: 10 April 2018 402.80 to 3912.66 . The highest intra-cluster distance was exhibited by cluster I (421.27) followed by cluster VI (316.27) and III (315.69). The highest inter cluster distance was observed between cluster II and VII (3912.66) followed by cluster II and VII (3831.40), cluster III and VI (2804.51) and cluster IV and VII. The above results indicate that these genotypes have maximum genetic diversity and useful for developing a large number of segregants through crossing programme by using maximum diverse genotypes. The genotypes of cluster VII showed the highest mean values for grain yield per plant, harvest index, biological yield per plant, primary and secondary branches per plant, plant height and days to maturity, while cluster VI showed the highest mean for grain yield per plot, total number of pods per plant, effective pods per plant and days to $50 \%$ flowering. The cluster V had highest mean value for 100 seed weight and canopy temperature at vegetative stage. The cluster IV recorded highest mean value for chlorophyll index, while cluster III exhibited highest mean value for canopy temperature at pollen formation stage. The promising genotypes for grain yield per plant, harvest index, biological yield per plant, grain yield per plot, total number of pods per plant, effective pods per plant, primary and secondary branches per plant, plant height, on the basis of mean values which could be utilized for hybridization programme for the development of high yielding genotypes. Among the fifteen traits studied, maximum contribution was made by 100 -seed weight $(46.83 \%)$ (Table-4) followed by total number of pods per plant $(22.22 \%)$, grain yield per plant $(12.54 \%)$, grain yield per plot $(5.34 \%)$ and harvest index $(3.49 \%)$. Therefore, these characters may be given importance during hybridization programme. 


\section{Introduction}

Chickpea (Cicer arietinum L.), a cool season crop is the largest produced food legume in South Asia and the third largest globally after common bean and field pea. India is the largest chickpea producer with $65 \%$ global production and chickpea constitutes about $40 \%$ of India's total pulse production. In spite of India being the largest chickpea producing country, a deficit exists in domestic production and demand which is met through imports. In India, the total area under chickpea is $8.25 \mathrm{M}$ ha with 7.33 million tonnes production with productivity $889 \mathrm{~kg} / \mathrm{ha}$ and the total area in Bihar reached 60.0 thousand ha with 57.50 thousand tonnes of production with productivity of $958 \mathrm{~kg} / \mathrm{ha}$ (2014-15: Agricultural Statistics Division, Directorate of Economics and Statistics, Dept. of Agriculture and Cooperation).

Limited or lack of genetic variability is important factor for the limited progress achieved in increasing the productivity of chickpea (Johnson et al., 2015). When the parents utilized in across are genetically similar, it is quite likely that the different lines derived reveals low diversity. On the contrary, when diverse parents are used in obtaining a segregating population, the derived lines reveal greater diversity despite sharing a common percentage. In any program aimed at genetic amelioration of yield, genetic diversity is the basic requirement. Effective hybridization program between genetically diverse parents will lead to considerable amount of heterotic response in $F_{1}$ hybrids and broad spectrum of variability in segregating generations. Mahalanobis's $\mathrm{D}^{2}$ statistics is a powerful tool in quantifying the degree of variability at the genotype level. Several workers studied the genetic diversity, clustering pattern, relative contribution of different characters toward divergence and effectiveness of selection (Patil et al., 2003;
Bisht et al., 2005; Johnson et al., 2015). So, the present experiment was formulated to study the genetic divergence and clustering pattern of the chickpea genotypes for selection of suitable parents for utilizing in hybridization programme and to study the genetic parameters attributing to yield.

\section{Materials and Methods}

The experimental material consisted of thirty six chickpea genotypes were sown on $10^{\text {th }}$ November, 2016 at Pulses Research farm, Bhitti, Bihar Agricultural University, Sabour (Bhagalpur). The experiment was laid out in a randomized complete block design with three replications during Rabi 2016-17 under All India Coordinated Research Project on Chickpea. The plot size was $4.8 \mathrm{~m}^{2}$, with 1 row of $4 \mathrm{~m}$ length. Inter row spacing distance was kept $30 \mathrm{~cm}$ and plant to plant spacing was $30 \times 10 \mathrm{~cm}$. The recommended packages of practices were followed to raise a healthy crop.

Data were recorded on fifteen quantitative traits viz. days to $50 \%$ flowering, days to maturity, chlorophyll index, canopy temperature at vegetative stage, canopy temperature at pollen formation stage, primary branches per plant, secondary branches per plant, plant height $(\mathrm{cm})$, total number of pods per plant, effective pods per plant, 100 seed weight (g), grain yield per plant $(\mathrm{g})$, biological yield per plant, harvest index and grain yield per plot $(\mathrm{g})$.The days to $50 \%$ flowering, days to maturity, and grain yield per plot were recorded on a plot basis and plant height and number of pods per plant and 100-seed weight were recorded from a random sample of five plants in each plot. Genetic divergence was estimated by using $\mathrm{D}^{2}$ statistics of Mahalanobis (1936) and clustering of genotypes was done according Ward minimum variance method as suggested by Ward (1963). The per cent contribution of 
characters towards genetic divergence was calculated according to Singh and Chaudhary (1985).

\section{Results and Discussion}

All the genotypes were grouped into seven clusters as per Ward minimum variance method, with cluster II containing the maximum of 13 genotypes followed by 9 genotypes in cluster I, 5 genotypes in cluster III, 3 genotypes each in cluster IV and V, 2 genotypes in cluster in cluster VI and monogenotypes in cluster VII (Table 1 and Fig. 1). The pattern of distribution of 36 genotypes in various clusters revealed existence of considerable genetic diversity in the material. It means the overall genetic similarity was found in the germplasms were presented within the cluster and the pattern of distribution of genotypes in different clusters exhibited that geographical diversity was not related to genetic diversity as genotypes of same geographical region were grouped into different cluster and vice-versa, as supported by earlier finding of Raval and Dobariya (2004), Parameshwarappa et al., 2011, Parashi et al., 2013, Kuldeep et al., 2015 and Kuldeep et al., 2015. The possible reason for grouping of genotypes of different places into one cluster could be free exchange of germplasm among the breeder of different region or unidirectional selection practiced by breeder in tailoring the promising cultivar for selection of different region. The intra-cluster $\mathrm{D}^{2}$ value ranged from 0.00 to 421.27 while, inter-cluster D value ranged from 402.80 to 3912.66 (Table 2 and Fig. 2) in Ward minimum variance method. The highest intra-cluster distance was exhibited by cluster I (421.27) followed by cluster VI (316.27) and III (315.69). The intra cluster distance was maximum in cluster I followed by cluster VI which indicated that hybridization involving genotypes within the same clusters may result in cross combination. The highest inter cluster distance was observed between cluster II and VII (3912.66) followed by cluster II and VII (3831.40), cluster III and VI (2804.51) and cluster IV and VII. These lines may be utilized in further breeding programme for the exploitation of hybrid vigour and suggesting wide diversity between them and genotypes in these clusters could be used as parents in hybridization programme to develop desirable type because crosses between genetically divergent lines will generate heterotic segregants. As heterosis can be best exploited and chances of getting transgressive segregants are maximum when generating diverse lines are crossed (Lal et al., 2011). Therefore, crosses between the members of clusters separated by inter-cluster distances are likely seemed to be beneficial for further improvement. Significant differences among the genotypes for different characters indicated variations among the genotypes favorable for their use in the breeding programs. Crosses between parents with maximum divergence would be more responsive to improvement since they are likely to produce higher heterosis and desirable genetic recombination (Kuldeep et al., 2015). The genotypes of cluster VII showed the highest mean values (Table 3 ) for grain yield per plant, harvest index, biological yield per plant, primary and secondary branches per plant, plant height and days to maturity, while cluster VI showed the highest mean for grain yield per plot, total number of pods per plant, effective pods per plant and days to $50 \%$ flowering. The cluster $\mathrm{V}$ had highest mean value for 100 seed weight and canopy temperature at vegetative stage. The cluster IV recorded highest mean value for chlorophyll index, while cluster III exhibited highest mean value for canopy temperature at pollen formation stage. The promising genotypes for grain yield per plant, harvest index, biological yield per plant, grain yield per plot, total number of pods per plant, effective pods per plant, primary and secondary branches per plant, plant height, on 
the basis of mean values which could be utilized for hybridization programme for the development of high yielding genotypes. Similar results were reported by Garje et al., (2013), Meshram et al., (2013), Nagy et al., (2013) and Singh et al., (2014). Among the fifteen traits studied, maximum contribution was made by 100 -seed weight $(46.83 \%)$ (Table 4) followed by total number of pods per plant $(22.22 \%)$, grain yield per plant (12.54\%), grain yield per plot $(5.34 \%)$ and harvest index (3.49\%). Therefore, these characters may be given importance during hybridization programme. On the basis of divergence classes the potential parent's viz. PhuleG1310, BRC-4, JG16, KPG 59, KWR 108, PG170, GNG2304, GNG2299, PG186, GNG 2264, GL29098, BG372, CSJ887, GCP105 can be used in the hybridization programme for chickpea improvement to obtain better transgressive segregants. This finding are in accordance with that of Wadikar et al., (2010), Akthar et al., (2011), Parameshwarappa et al., (2011), Jayalakshmi et al., (2012), Puri et al., (2013), Gaikwad et al., (2014) and Johnson et al., (2015). The high inter cluster distance was observed between clusters, suggesting wide diversity between them and genotypes in these clusters could be used as parents in hybridization programme to develop high yielding chickpea genotypes under normal sown condition because crosses between genetically divergent lines will generate heterotic sergeants. Among the fifteen traits studied, maximum contribution was made by plot yield followed by plant stand at harvest and number of pods per plant.

Table.1 Distribution of 36 chickpea genotypes in various clusters

\begin{tabular}{|c|c|l|}
\hline Cluster & $\begin{array}{l}\text { No. of } \\
\text { Genotypes }\end{array}$ & \multicolumn{1}{|c|}{ Name of Genotypes } \\
\hline I & 09 & $\begin{array}{l}\text { JG 74315-14, BRC-3, IPC 2012-49, BG 3067, IPC 2012-49, } \\
\text { GL12003, GNG 2207, H12-62, H12-55 }\end{array}$ \\
\hline II & 13 & $\begin{array}{l}\text { BRC-4, JG16, KPG 59, KWR 108, PG170, GNG2304, GNG2299, } \\
\text { PG186, GNG 2264, GL29098, BG372, CSJ887, GCP105 }\end{array}$ \\
\hline III & 05 & IPC2012-98, PBC501, DCP92-3, NDG14-24, BG3068 \\
\hline IV & 03 & GNG469,BG3043, GNG2215 \\
\hline V & 03 & NBeG507, ICCV15112, JG24 \\
\hline VI & 02 & BRC-2, BRC-1 \\
\hline VII & 01 & PhuleG1310 \\
\hline
\end{tabular}

Table.2 Average intra and inter cluster distance values among eight clusters for thirty six genotypes of chickpea under normal condition

\begin{tabular}{|c|r|r|r|r|r|r|r|}
\hline Cluster & I & II & III & IV & \multicolumn{1}{|c|}{ V } & \multicolumn{1}{|c|}{ VI } & \multicolumn{1}{c|}{ VII } \\
\hline I & 421.723 & 509.565 & 827.818 & 1186.26 & 815.841 & 1221.331 & 2571.000 \\
\hline II & & 175.988 & 402.805 & 1254.806 & 1533.432 & 2123.469 & 3912.661 \\
\hline III & & & 315.619 & 768.203 & 1698.819 & 2804.511 & 3831.403 \\
\hline IV & & & & 223.515 & 1102.483 & 2517.610 & 2486.896 \\
\hline V & & & & & 147.343 & 902.239 & 1387.935 \\
\hline VI & & & & & & 316.279 & 1873.666 \\
\hline VII & & & & & & & 0.000 \\
\hline
\end{tabular}


Table.3 Mean values of clusters of different characters towards genetic divergence in thirty six chickpea genotypes under normal sown condition

\begin{tabular}{|c|c|c|c|c|c|c|c|c|c|c|c|c|c|c|c|}
\hline $\begin{array}{l}\frac{2}{0} \\
\frac{0}{0} \\
\frac{0}{0}\end{array}$ & 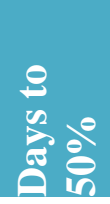 & 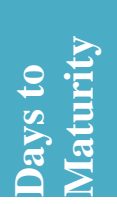 & 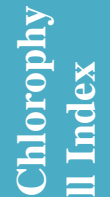 & 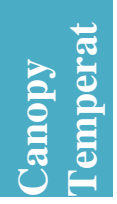 & 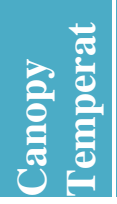 & 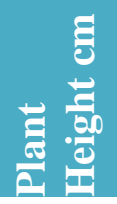 & 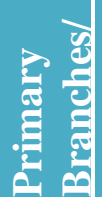 & 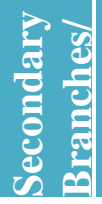 & 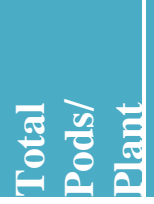 & 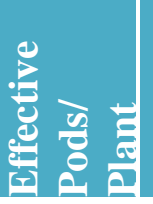 & $\begin{array}{l}\overline{\mathscr{J}} \\
\frac{0}{00} \\
\frac{0}{0} \\
0\end{array}$ & 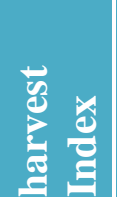 & 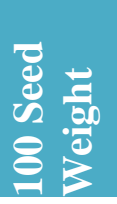 & $\frac{\stackrel{\vec{E}}{\frac{\vec{E}}{2}}}{\frac{0}{\sigma}}$ & 苞尊 \\
\hline I & $\begin{array}{c}79.5 \\
00\end{array}$ & $\begin{array}{c}133.8 \\
89\end{array}$ & $\begin{array}{c}63.2 \\
59\end{array}$ & $\begin{array}{c}22.2 \\
19\end{array}$ & $\begin{array}{c}33.7 \\
67\end{array}$ & $\begin{array}{c}64.66 \\
7\end{array}$ & $\begin{array}{l}1.7 \\
56\end{array}$ & $\begin{array}{l}4.6 \\
44\end{array}$ & 78.521 & 71.216 & 83.987 & $\begin{array}{c}24.6 \\
15\end{array}$ & $\begin{array}{c}21.97 \\
2\end{array}$ & $\begin{array}{c}20.74 \\
7\end{array}$ & $\begin{array}{c}2157.51 \\
1\end{array}$ \\
\hline II & $\begin{array}{c}79.2 \\
31\end{array}$ & $\begin{array}{c}133.5 \\
38\end{array}$ & $\begin{array}{c}61.0 \\
13\end{array}$ & $\begin{array}{c}22.0 \\
60\end{array}$ & $\begin{array}{c}34.4 \\
90\end{array}$ & $\begin{array}{c}56.13 \\
5\end{array}$ & $\begin{array}{l}1.5 \\
38\end{array}$ & $\begin{array}{l}4.0 \\
54\end{array}$ & 61.654 & 54.058 & 48.531 & $\begin{array}{c}23.3 \\
74\end{array}$ & $\begin{array}{c}16.75 \\
0\end{array}$ & $\begin{array}{c}11.09 \\
6\end{array}$ & $\begin{array}{c}2164.88 \\
9\end{array}$ \\
\hline III & $\begin{array}{c}76.5 \\
00\end{array}$ & $\begin{array}{c}133.2 \\
00\end{array}$ & $\begin{array}{c}61.0 \\
30\end{array}$ & $\begin{array}{c}20.7 \\
90\end{array}$ & $\begin{array}{c}35.0 \\
40\end{array}$ & $\begin{array}{c}56.59 \\
0\end{array}$ & $\begin{array}{l}1.6 \\
00\end{array}$ & $\begin{array}{l}4.5 \\
00\end{array}$ & 43.084 & 33.128 & 78.606 & $\begin{array}{c}20.3 \\
46\end{array}$ & $\begin{array}{c}18.15 \\
0\end{array}$ & $\begin{array}{c}15.24 \\
6\end{array}$ & $\begin{array}{c}2030.40 \\
4\end{array}$ \\
\hline IV & $\begin{array}{c}78.5 \\
00\end{array}$ & $\begin{array}{c}132.8 \\
33\end{array}$ & $\begin{array}{c}65.0 \\
53\end{array}$ & $\begin{array}{c}21.7 \\
08\end{array}$ & $\begin{array}{c}34.3 \\
58\end{array}$ & $\begin{array}{c}63.36 \\
7\end{array}$ & $\begin{array}{l}1.9 \\
33\end{array}$ & $\begin{array}{l}4.4 \\
00\end{array}$ & 79.793 & 63.495 & 134.573 & $\begin{array}{c}19.0 \\
10\end{array}$ & $\begin{array}{c}28.00 \\
0\end{array}$ & $\begin{array}{c}24.94 \\
7\end{array}$ & $\begin{array}{c}2357.50 \\
2\end{array}$ \\
\hline $\mathbf{V}$ & $\begin{array}{c}77.1 \\
67\end{array}$ & $\begin{array}{c}135.3 \\
33\end{array}$ & $\begin{array}{c}64.7 \\
87\end{array}$ & $\begin{array}{c}22.3 \\
42\end{array}$ & $\begin{array}{c}34.2 \\
33\end{array}$ & $\begin{array}{c}70.73 \\
3\end{array}$ & $\begin{array}{l}1.6 \\
00\end{array}$ & $\begin{array}{l}4.1 \\
33\end{array}$ & & & & $\begin{array}{c}18.3 \\
17\end{array}$ & $\begin{array}{c}37.75 \\
0\end{array}$ & $\begin{array}{c}17.06 \\
3\end{array}$ & $\begin{array}{c}1764.30 \\
2\end{array}$ \\
\hline VI & $\begin{array}{c}80.7 \\
50\end{array}$ & $\begin{array}{c}135.5 \\
00\end{array}$ & $\begin{array}{c}63.8 \\
65\end{array}$ & $\begin{array}{c}21.9 \\
13\end{array}$ & $\begin{array}{c}34.2 \\
25\end{array}$ & $\begin{array}{c}65.25 \\
0\end{array}$ & $\begin{array}{l}1.9 \\
00\end{array}$ & $\begin{array}{l}4.3 \\
00\end{array}$ & $\begin{array}{c}163.74 \\
7\end{array}$ & $\begin{array}{c}158.57 \\
0\end{array}$ & 109.540 & $\begin{array}{c}19.8 \\
47\end{array}$ & $\begin{array}{c}26.25 \\
0\end{array}$ & $\begin{array}{c}21.38 \\
5\end{array}$ & $\begin{array}{c}2449.59 \\
7\end{array}$ \\
\hline VII & $\begin{array}{c}76.0 \\
00\end{array}$ & $\begin{array}{c}138.0 \\
00\end{array}$ & $\begin{array}{c}62.7 \\
70\end{array}$ & $\begin{array}{c}22.1 \\
75\end{array}$ & $\begin{array}{c}34.4 \\
75\end{array}$ & $\begin{array}{c}67.00 \\
0\end{array}$ & $\begin{array}{l}2.6 \\
00\end{array}$ & $\begin{array}{l}5.6 \\
00\end{array}$ & 77.220 & 72.518 & 316.800 & $\begin{array}{c}24.7 \\
80\end{array}$ & $\begin{array}{c}31.75 \\
0\end{array}$ & $\begin{array}{c}77.22 \\
0\end{array}$ & $\begin{array}{c}1808.04 \\
5\end{array}$ \\
\hline Mean & $\begin{array}{c}78.6 \\
81\end{array}$ & $\begin{array}{c}133.9 \\
03\end{array}$ & $\begin{array}{c}62.4 \\
35\end{array}$ & $\begin{array}{c}21.9 \\
12\end{array}$ & $\begin{array}{c}34.3 \\
38\end{array}$ & $\begin{array}{c}60.95 \\
8\end{array}$ & $\begin{array}{l}1.6 \\
89\end{array}$ & $\begin{array}{l}4.3 \\
56\end{array}$ & 70.565 & 62.373 & 82.558 & $\begin{array}{c}22.3 \\
22\end{array}$ & $\begin{array}{c}21.88 \\
2\end{array}$ & $\begin{array}{c}18.14 \\
5\end{array}$ & $\begin{array}{c}2132.93 \\
9\end{array}$ \\
\hline $\begin{array}{l}\text { TreatM } \\
\text { SS }\end{array}$ & $\begin{array}{c}11.2 \\
95\end{array}$ & 7.131 & $\begin{array}{c}16.7 \\
27\end{array}$ & $\begin{array}{c}1.63 \\
5\end{array}$ & $\begin{array}{c}1.15 \\
7\end{array}$ & $\begin{array}{c}179.8 \\
10\end{array}$ & $\begin{array}{l}0.2 \\
99\end{array}$ & $\begin{array}{l}0.7 \\
50\end{array}$ & $\begin{array}{c}4710.3 \\
43\end{array}$ & $\begin{array}{c}4963.4 \\
62\end{array}$ & $\begin{array}{c}15919.7 \\
22\end{array}$ & $\begin{array}{c}36.1 \\
11\end{array}$ & $\begin{array}{c}283.0 \\
61\end{array}$ & $\begin{array}{c}880.4 \\
02\end{array}$ & $\begin{array}{c}187269 \\
525\end{array}$ \\
\hline ErrMSS & $\begin{array}{c}11.7 \\
37\end{array}$ & $\begin{array}{c}10.09 \\
2\end{array}$ & $\begin{array}{c}8.53 \\
6\end{array}$ & $\begin{array}{c}1.60 \\
7\end{array}$ & $\begin{array}{c}3.78 \\
6\end{array}$ & $\begin{array}{c}93.45 \\
2\end{array}$ & $\begin{array}{c}0.1 \\
10\end{array}$ & $\begin{array}{l}0.7 \\
86\end{array}$ & $\begin{array}{c}388.45 \\
2\end{array}$ & $\begin{array}{c}348.65 \\
2\end{array}$ & 559.886 & $\begin{array}{c}20.0 \\
36\end{array}$ & $\begin{array}{c}17.20 \\
9\end{array}$ & $\begin{array}{c}52.88 \\
9\end{array}$ & $\begin{array}{c}208367 . \\
304\end{array}$ \\
\hline F Ratio & $\begin{array}{c}0.96 \\
2\end{array}$ & 0.707 & $\begin{array}{c}1.96 \\
0\end{array}$ & $\begin{array}{c}1.01 \\
7\end{array}$ & $\begin{array}{c}0.30 \\
6\end{array}$ & 1.924 & $\begin{array}{l}2.7 \\
20\end{array}$ & $\begin{array}{l}0.9 \\
54\end{array}$ & 12.126 & 14.236 & 28.434 & $\begin{array}{c}1.80 \\
2\end{array}$ & $\begin{array}{c}16.44 \\
9\end{array}$ & $\begin{array}{c}16.64 \\
6\end{array}$ & 0.899 \\
\hline $\begin{array}{l}\text { Probabil } \\
\text { ity }\end{array}$ & $\begin{array}{c}0.45 \\
6\end{array}$ & 0.623 & $\begin{array}{c}0.11 \\
4\end{array}$ & $\begin{array}{c}0.42 \\
5\end{array}$ & $\begin{array}{c}0.90 \\
6\end{array}$ & 0.120 & $\begin{array}{l}0.0 \\
38\end{array}$ & $\begin{array}{l}0.4 \\
61\end{array}$ & 0.000 & 0.000 & 0.000 & $\begin{array}{c}0.14 \\
3\end{array}$ & 0.000 & 0.000 & 0.495 \\
\hline
\end{tabular}


Table.4 Contribution of different characters towards genetic divergence of 36 chickpea genotypes

\begin{tabular}{|c|c|c|c|}
\hline SI. No. & Source & Contribution \% & Contribution \% \\
\hline 1. & Days to $50 \%$ Flowering & 0.16 & 1.000 \\
\hline 2. & Days to Maturity & 0.16 & 1.000 \\
\hline 3. & Chlorophyll Index & 0.01 & 0.000 \\
\hline 4. & Canopy Temperature@vs & 0.32 & 2.000 \\
\hline 5. & Canopy Temperature @pfs & 0.01 & 0.000 \\
\hline 6. & Plant Height (cm) & 3.02 & 19.000 \\
\hline 7. & Primary Branches/ Plant & 1.59 & 10.000 \\
\hline 8. & Secondary Branches/ Plant & 0.16 & 1.000 \\
\hline 9. & Total number of Pods/ Plant & 22.22 & 140.000 \\
\hline 10. & Effective Pods/ Plant & 2.22 & 14.000 \\
\hline 11. & Biological Yield per plant (g) & 1.90 & 12.000 \\
\hline 12. & Harvest Index (\%) & 3.49 & 22.000 \\
\hline 13. & 100 Seed Weight (g) & 46.83 & 295.000 \\
\hline 14. & Grain Yield/ Plant (g) & 12.54 & 79.000 \\
\hline 15. & Grain Yield/ plot (kg/ha) & 5.34 & 34.000 \\
\hline
\end{tabular}

Fig.1 Ward minimum variance dendogram

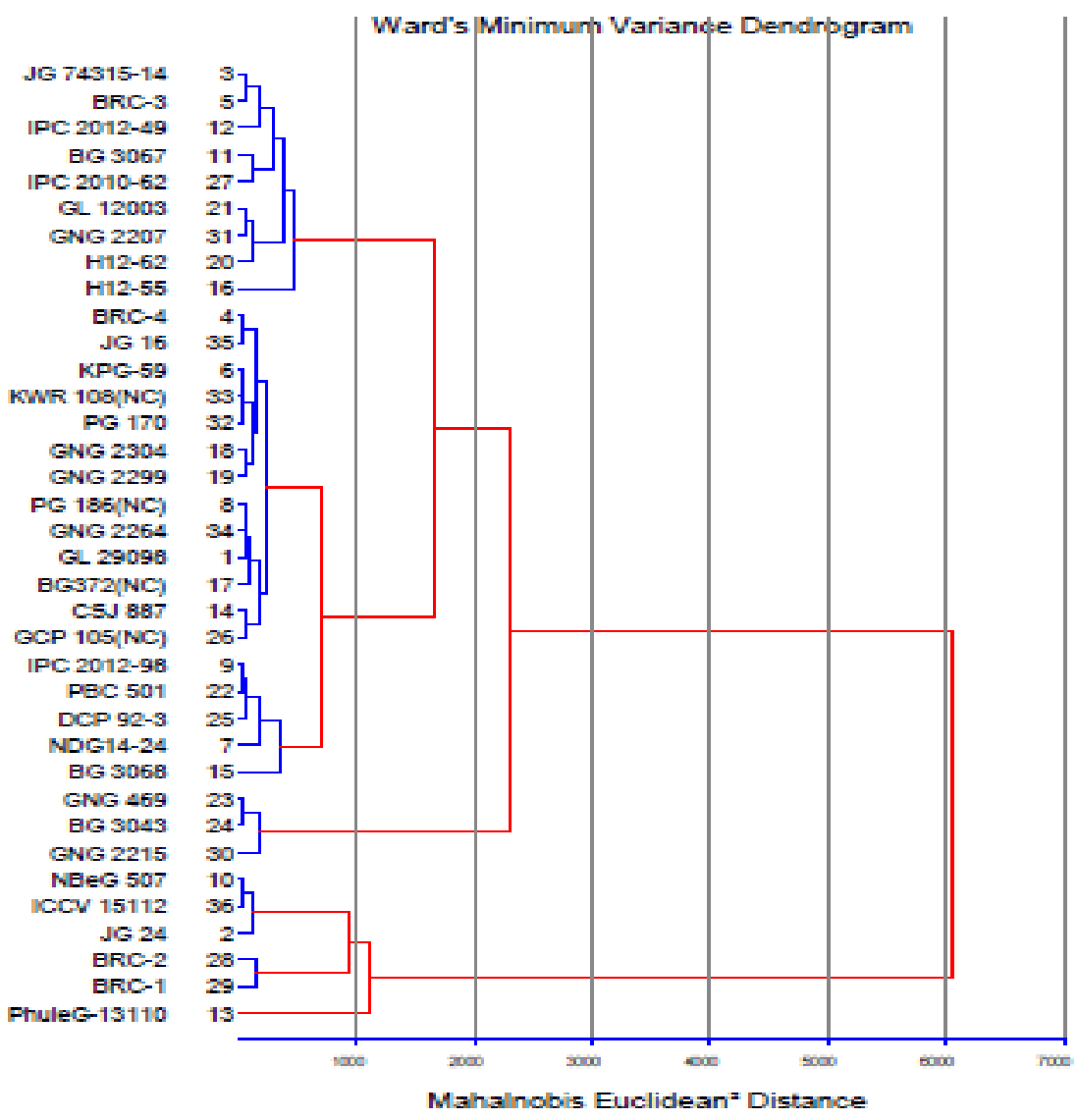


Fig.2 Euclidean ${ }^{2}$ distance

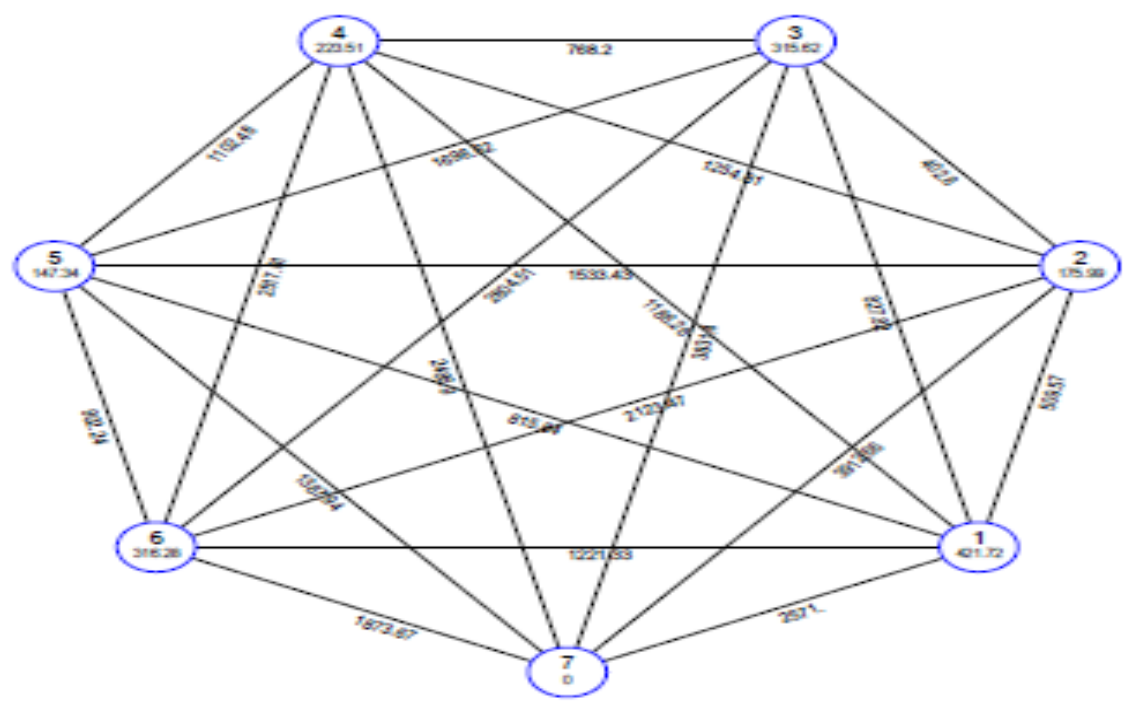

Euclidean² Distance (Not to the Scale)

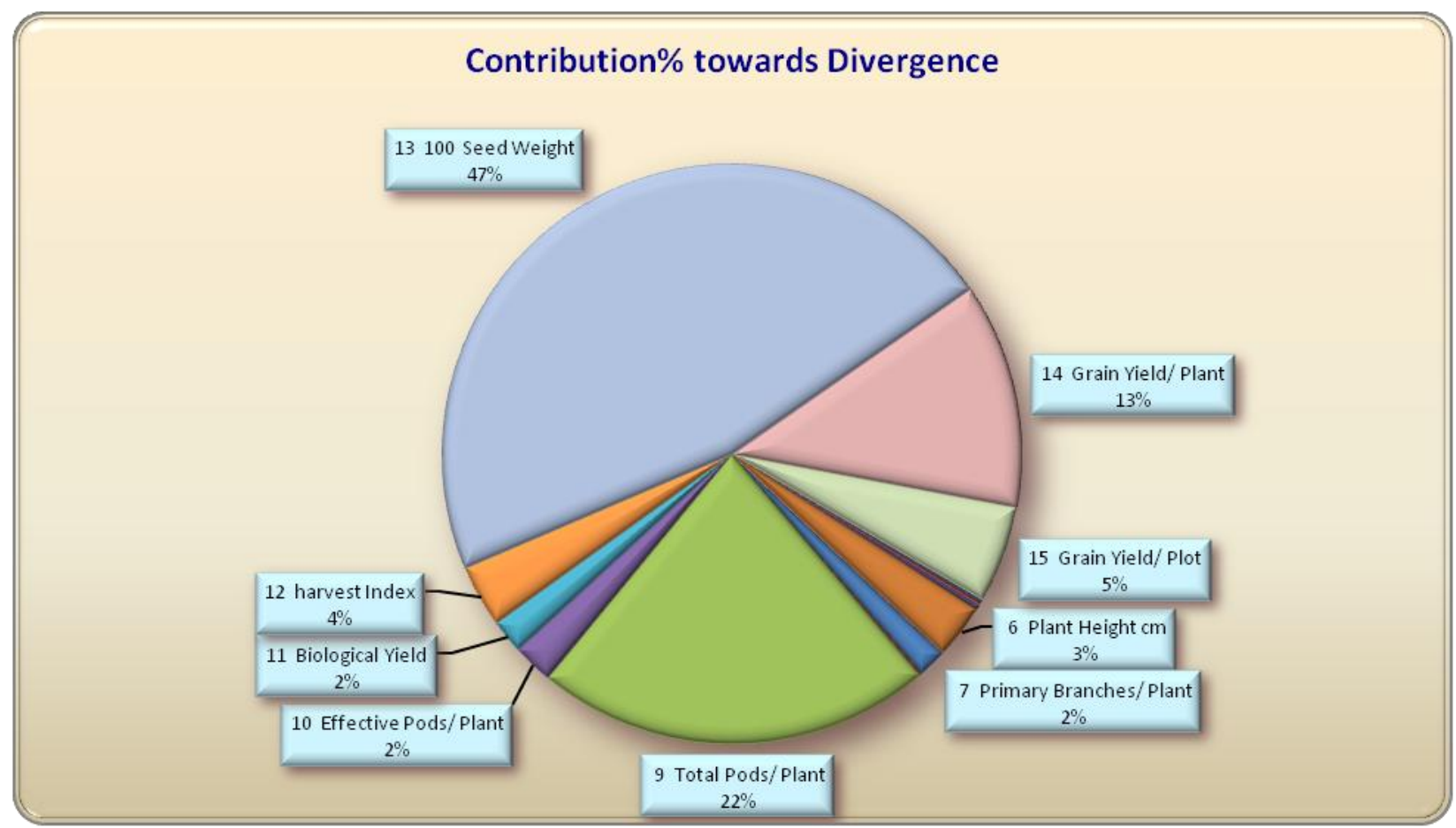

Therefore, these characters may be given importance during hybridization programme and offers provides the opportunity for breeding programs to improve the chickpea leading to better yield stability and profitability for growers. The above findings, thus, would be useful for planning a comprehensive hybridization programme for obtaining desirable segregants for selection of high yielding chickpea genotypes under normal sown condition. 
In the present investigation, high inter cluster distance was observed between clusters, suggesting that there are a wide diversity between them and genotypes in these clusters could be used as parents in hybridization programme to develop high yielding chickpea genotypes under normal sown condition. The above results indicate that these genotypes have maximum genetic diversity and useful for developing a large number of segregants through crossing programme by using maximum diverse genotypes. Considering the mean performance of genotypes, diversity of clusters they belonged and cluster means for different characters, hybridization of BRC-4, JG16, KPG 59, KWR 108, PG170,GNG2304, GNG2299, PG186, GNG 2264, GL29098, BG372, CSJ887, GCP105 of cluster II with promising genotype PhuleG1310 of cluster VII may be recommended for isolating transgressive segregants for developing high yielding rice varieties. Intercrossing of divergent genotypes with desirable traits would lead to greater opportunity for exploitation of maximum amount of heterosis and utilize them for multiple crossing programmes.

\section{References}

Akhtar, L. H., Muhammad, A. and Muhammad, N. 2011. Genetic divergence and inter-relationship studies in chickpea (Cicer arietinum L.). Pak. J. Agril. Sci. 48(1): 35-39.

Bisht, I. S., Bhat, K. V., Lakhanpaul, S., Latha, M., Jayan, P. K., Biswas, B. K. and Singh, A. K. 2005. Diversity and genetic resources of wild vigna species in India. Genet. Resource. Crop. Evol. 52: $53-68$

Gaikwad, A. R., Desai, N. C., Pawar, G. H and Langhi, A. M. 2014. Genetic divergence in chickpea. International $\mathrm{J}$. Agril. Sci. 10(2): 812- 815.
Garje, U. A., Bhailume, M. S. and Nagawade, D. R. 2013. Genetic diversity analysis of green gram (Vigna radiate L. Wilczek.). The Bioscan. 8(4): 14771480.

Jayalakshmi, V., Reddy, C. K. K. and Jyothirmayi, G. 2012. Genetic study on drought tolerance attributes in Chickpea (Cicer arietinum L.). J. Food Legumes. 25: 94-96.

Johnson P.L., Sharma R.N. and Nanda H.C. 2015. Genetic diversity and association analysis for yield traits chickpea (Cicer arietinum L.) under rice based cropping system. The Bioscan 10(2): 879-884.

Kuldeep RK, Pandey S, Babbar A and Mishra DK 2014. Genetic variability, character association and path coefficient analysis in chickpea grown under heat stress condition. Electronic Journal of Plant Breeding 5(4):812-819.

Kuldeep RK, Pandey S, Babbar A and Prakash V 2015. Genetic diversity analysis in chickpea grown under heat stress condition. Electronic Journal of Plant Breeding, 6(2): 424-433.

Lal, D., Krishna, R. and Gurpreet, S. 2001. Genetic divergence in chickpea. Ind. J. Pulses Res., 14 (1): 63-64.

Mahalanobis, P. C. 1936. On generalized distance in statistics. Proceed. National Institute of Sci., 2: 49-55.

Meshram, M. P., Ali, R. I., Patil A. N. And Meena, Sunita. 2013. Variability studies in $\mathrm{m} 3$ generation in blackgram (Vigna mungo (L.) Hepper). The Bioscan. 8(4): 1357-1361.

Nagy, K. and Sharma, R. N. and Nanda, H. C. and Kanwar, S. S. (2013). Genetic Variability and Association Studies among Yield Attributes in Pigeonpea [Cajanus cajan (L.) Millsp.] Accessions of Bastar. The Ecoscan. 4(Spl): $267-$ 271.

Parameshwarappa, S. G., Salimath P. M., Upadhyaya U. D., Patil, S. S. and 
Kajjidoni, S. T. 2011. Genetic divergence under three environments in a minicore collection of Chickpea (Cicer arietinum L). Indian J. Plant Genetic Resources 24: 177-185.

Parashi, V.S., Lad, D.B., Mahse, L.B., Kute, N.S. and Sonawane, C.J. 2013. Genetic diversity studies in chickpea (Cicer arietinum L.), Bioinfolet, 10 (1): 337341.

Patil, B. L., Hegde V. S. and Salimath P. M. 2003. Studies on genetic divergence over stress and non-stress environment in mung bean. Indian J. Genet. 63: 7778.

Puri, M. K., Johnson P. L. and Sharma R. N. 2013. Study of variability, diversity and association analysis of chickpea (Cicer arietinum L.) germplasm under normal and late sown condition of Chhattisgarh state. Trends Biosc. 6(6): 723-731.

Rao, C R 1952. Advanced Statistical Methods in Biometrical Research John Wiley and Sons, New York. P.351.

Raval, L.J. and Dobariya, K. 2004. Assessment of genetic divergence in chickpea (Cicer arietinum L.). Ann. Agric. Res., 25 (1): 30-34.

Singh, R. K. and Chaudhary, B. D. 1977. Biometrical methods in quantitative genetic analysis. Kalyani Publishers, New Delhi. pp 266.

Wadikar, P. B., Ghodke, M. K. and Pole, S. P. 2010. Genetics divergence for productivity traits in chickpea (Cicer arietinum L.). J. Food Legumes. 23: $245-246$.

\section{How to cite this article:}

Tejashwini Agrawal, Anand Kumar, Sanjay Kumar, Anil Kumar, Manoj Kumar, Satyendra and Sadia Perween. 2018. Assessment of Genetic Diversity in Chickpea (Cicer arietinum L.) Germplasm under Normal Sown Condition of Bihar. Int.J.Curr.Microbiol.App.Sci. 7(04): 3552-3560. doi: https://doi.org/10.20546/ijcmas.2018.704.400 